\title{
Zwischen Wunsch und Realität: Ausbildungs- und Berufsverläufe von jungen Frauen
}

\section{Evéline Huber und Manfred Max Bergman'1}

Dieser Artikel hat zum Ziel, den Einfluss von signifikanten Anderen auf Wunschberufe und tatsächlich ausgeübte Berufe von jungen Frauen zu untersuchen. Junge Frauen zwischen Jahrgang 1983 und 1989 wurden bezüglich ihrer Wunschberufe und ihren tatsächlich ausgeübten Berufen interviewt. Mittels qualitativer Inhaltsanalyse wurden vier Gruppen von Akteuren identifiziert, die unterschiedliche Einflüsse auf die jungen Frauen ausübten. Die Resultate zeigen, dass eine Vergeschlechtlichung insbesondere in Familien, dort vor allem von Seiten der Mutter, festzustellen ist. Während Mütter als emotionell-fördernd wahrgenommen werden, erscheinen Väter eher als Ansprechperson in Bezug auf die Finanzierung von Weiterbildungen. Typisch weibliche Berufe wie (Kindergarten-)Lehrerin gelten im frühen Kindesalter oft als Traumberuf, was bereits als vergeschlechtlichende Weichenstellung verstanden werden kann.

\section{Einleitung}

Die Erwerbsarbeit weist unterschiedliche Formen geschlechtsspezifischer Strukturierungen auf. Ausbildungs- und Berufsverläufe werden einerseits durch Institutionen, andererseits durch Wert- und Normvorstellungen mitgeprägt. Sie unterliegen aber gleichzeitig auch individuellen Wünschen und strukturellen Möglichkeiten. Dies zeigt sich in der Lohndifferenz, bei der Vergabe von Kader- oder Führungspositionen sowie bei der Vereinbarkeit von Familie und Beruf (Strub, Hüttner und Guggisberg, 2005). Die Konzentration von Frauen in bestimmten Berufsgruppierungen ist demnach die Konsequenz von erlebten oder antizipierten geschlechtsspezifischen Barrieren, aber auch persönlichen Wünschen (Borkowski, 2000). Die bisherige Forschung konnte zeigen, dass subjektive, institutionelle und (geschlechter-)normative Einflussfaktoren zur beruflichen Segregation und einer Benachteiligung von Frauen beitragen (u. a. Baumgartner \& Fux, 2004; Leemann \& Keck, 2005). Zudem führt insbesondere das duale Bildungssystem der Schweiz zu Zuweisungen in «typisch männliche» 
oder "typisch weibliche» Berufe (Imdorf, 2004; Imdorf et al., 2010; Bergman et al., 2011, 2012), was den Zugang zu geschlechtsuntypischen Berufsfeldern erschwert (Leemann \& Keck, 2005, S. 72 ff.). Der berufsbiographische Verlauf ist jedoch nicht als eine Ansammlung punktueller Entscheidungen zu deuten, sondern als längerer Prozess, der eine komplexe Verschränkung verschiedener Faktoren und Mechanismen aufweist (Maihofer, Bergman, Huber, Hupka, Kanji, Samuel, Schwiter und Wehner, 2012). Cornelissen und Gille (2005) zeigen, dass bereits junge Kinder «Traumberufe» nennen, die aufgrund ihrer sozialisatorisch erworbenen Wert- und Normvorstellungen geschlechtstypisch sind, wobei generell «Frauenberufe» ${ }^{2}$ als weniger wünschenswert gelten als «Männerberufe». Viele Frauen fühlen sich auch den Anforderungen eines typischen Männerberufes nicht gewachsen (Cornelissen, 2009). Die bisherige Forschung konnte zeigen, dass positive Rückmeldungen wichtig sind, um sich in einer Berufssituation sicher zu fühlen und eine berufliche Identität zu entwickeln. Fehlen diese Rückmeldungen oder fallen sie negativ aus, kann es zu einem grundsätzlichen Infragestellen des eingeschlagenen Berufsweges führen (Schwiter, Wehner, Maihofer und Huber, 2011). Zudem entscheiden sich Frauen nach wie vor überdurchschnittlich oft für kürzere Ausbildungen als Männer und verbauen sich dadurch den Zugang zu weiteren Qualifikationsmöglichkeiten (Baumgartner \& Fux, 2004). Generell stellen Bildungsentscheide gesteigerte Anforderungen an Jugendliche durch eine Optionsvielfalt dieser Generation, durch Ausdifferenzierung von Berufen, aber auch durch eine grössere geografische Mobilität. Individuelle Wünsche und strukturelle Möglichkeiten, aber auch Erwartungen der Eltern müssen miteinander vereinbart werden. Viele stehen deshalb vor der Schwierigkeit, ihre eigenen Wünsche und Bedürfnisse von denen der Eltern oder weiterer signifikanter Anderer abzugrenzen. Dadurch kann ein Spannungsfeld zwischen finanzieller und emotionaler Abhängigkeit entstehen. Um das Spannungsfeld zwischen eigener Entscheidungsfindung und den Erwartungen signifikanter Anderer bei jungen Frauen näher beleuchten zu können, wird in diesem Artikel untersucht, welche signifikante Andere überhaupt einen Einfluss auf die jungen Frauen ausüben, wie sich ihr Einfluss genau zeigt und welche vergeschlechtlichende Prozesse während der bisherigen lebensbiografischen Verläufe gewirkt haben.

\section{Theoretische Rahmung}

In der Sozialisation sind insbesondere Eltern und Grosseltern im frühen Alter, später gute FreundInnen, LehrerInnen und andere Vertraute, im späteren Lebensverlauf dann auch (Ehe-)PartnerInnen oder LebensgefährtInnen bedeutende signifikante Andere (Faltermeier, 2001). Zudem gelten Institutionen wie Kindergarten und Schulen als wichtige Orte der sozio-kulturellen Orientierung und Identitätsfindung (Bourdieu \& Passeron, 1971). Sowohl die schulische 
Bildung als auch die familiäre Sozialisation habitualisieren Kinder und Jugendliche in ihrem Wahrnehmen, Denken und Handeln (Kalthoff, 2004). Dabei werden geschlechtsspezifische Wahrnehmungs-, Denk- und Handlungsschemata herausgebildet (Bourdieu, 2005), was dazu führt, dass potenzielle Möglichkeiten menschlicher Erlebnis- und Handlungsweisen verwehrt werden (Krais \& Gebauer, 2002). Die geschlechtsspezifische Sozialisation produziert und reproduziert soziale Differenzierungen und gelebte Geschlechterhierarchien. Zudem prägen Geschlechterstereotypen nicht nur Selbstbilder und Präferenzen, sondern zementieren scheinbar natürliche Differenzen bezüglich individueller Fähigkeiten und Aspirationsmöglichkeiten (Buchmann \& Kriesi, 2009; Charles 2005, 25ff. Cornelissen, 2009; Imdorf, 2004; Maihofer, 2002, 2004; Ostendorf, 2005). Für Bourdieu hängt die Herausbildung eines Geschlechterhabitus mit der vorherrschenden patriarchalen heteronormativen Gesellschafts- und Geschlechterordnung zusammen (Schwiter, Wehner, Maihofer \& Huber, 2011). Gesellschaftsverhältnisse geben eine Richtung für geschlechtsspezifische Sozialisationsprozesse vor, die bereits in frühster Kindheit eingeübt und verinnerlicht werden müssen. Die eingeübten Verhaltensweisen wirken sich in der Konsequenz auf die erwünschte und später ausgeübte Erwerbstätigkeit aus. Goffman (2001) zeigte, dass sich verinnerlichte Geschlechterdispositionen, also die Darstellung von Geschlecht, schliesslich in geschlechtsspezifischen institutionellen Zuweisungen, eben in einem institutionalisierten Genderismus, zeigt. Zuweisungen zu bestimmten geschlechtstypischen Berufen finden so eine Legitimierung. In den Institutionen wird auch erkennbar, dass geschlechtsspezifische Unterschiede in den Ausbildungs- und Berufswegen sowohl vorausgesetzt als auch reproduziert und weiter verstärkt werden (Becker, 2008; Born \& Krüger, 2001; Gottschall, 2009; Wetterer, 2002; Levy, 1977). Es ist als eine andauernde Sozialisationsarbeit anzusehen, sich als Individuum geschlechtlich zu differenzieren und möglichst einem geschlechtstypischen Ideal zu entsprechen (Bourdieu, 2005). Da dadurch junge Frauen und Männer bereits als Vergeschlechtlichte in Schule, Ausbildung und Beruf eintreten, werden entsprechend dem Versuch, dem Ideal gerecht zu werden, oft geschlechtstypische Verläufe bevorzugt (Jäger, König \& Maihofer, 2011). Sowohl die familiäre Sozialisation als auch Einflüsse von weiteren Akteuren ausserhalb der Familie sind entscheidend für geschlechtstypische oder -untypische Verläufe. Für den Ausbildungs- und Berufsverlauf ist also einerseits entscheidend, wer, andererseits aber auch wie sozialisiert wird. Insbesondere diejenigen Einflüsse, die nicht entlang geschlechtsspezifischer Pfadabhängigkeiten verlaufen, könnten demnach für einen neutralen oder sogar geschlechtsuntypischen Ausbildungs- und Berufsverlauf fördernd sein.

\section{Empirische Untersuchung}

In diesem Artikel wird untersucht, wie die Einflüsse signifikanter Anderer wahrgenommen werden und wie sie sich über eine der wichtigsten Lebensphasen 
junger Frauen entwickeln. Um den beschriebenen geschlechtersegregierenden Prozess darstellen zu können, sind leitfadengestützte Interviews geführt worden. Die Untersuchung umfasst insgesamt dreizehn junge Frauen zwischen den Jahrgängen 1983 und 1989 aus der Deutschschweiz. Die Pretests zu den Interviews fanden im Mai 2010 statt. Die Interviews wurden darauf zwischen Juni 2010 und April 2011 in der Deutschschweiz geführt. Die Auswahl der Interviewpartnerinnen erfolgte nach einem theoretischen Sampling. Die Identifizierung von signifikanten Anderen und deren Art der Beeinflussung in Bezug auf die Ausbildungs- und Berufsverläufe der jungen Frauen standen im Zentrum dieser Interviews. Die Analyse der Interviews erfolgte mittels qualitativer Inhaltsanalyse nach Green et al. (2007).

\section{Resultate}

Die Analyse identifizierte in einem ersten Schritt signifikante Andere, die Rolle der signifikanten Anderen im Ausbildungs- und Berufsverlauf der jungen Frauen und die konkrete Art ihres Einflusses. In einem zweiten Schritt wurden die Interviews unter den Aspekten der geschlechtsspezifischen Norm- und Wertvorstellungen der signifikanten Anderen analysiert, der damit verbunden Reproduktion geschlechtsspezifischer Ungleichheiten bei der Wahl und Ausübung der Berufe sowie der von den Frauen selbst wahrgenommenen Vergeschlechtlichung.

\section{Signifikante Andere}

Die qualitative Inhaltsanalyse der Interviewtranskripte ergab vier Hauptgruppen von signifikanten Anderen: Familienmitglieder, FreundInnen, LehrerInnen/ LehrmeisterInnen/Vorgesetzte und Andere (heterogene Gruppe). Vier Typen von wahrgenommenen Einflüssen konnten dabei identifiziert werden: fördernd, blockierend, beratend und hinnehmend. Exemplarisch werden nachfolgend die einzelnen Einflüsse innerhalb der oben genannten Hauptgruppen von signifikanten Anderen diskutiert.

\section{Familie}

In der Hauptgruppe Familie befinden sich die Eltern der jungen Frauen, (Ehe-) Partner und eigene Kinder, Geschwister und weitere Verwandte (z.B. Onkel und Tante).

Der fördernde Einfluss zeigt sich insbesondere daran, dass signifikante Andere explizit positiv bei der schulischen und beruflichen Entwicklung mitgewirkt haben. In der Familie von Veronika zeigt sich vor allem bei der Mutter ein fördernder Einfluss. Ihr erscheint es wichtig, dass die Kinder genügend institutionalisiertes Kapital in Form von Bildungstiteln (Bourdieu, 1983) anhäufen können. Veronikas Eltern, beide mit einem Abschluss auf Sek.-II-Stufe, fördern 
ihre drei Kinder in Richtung weiterführender Schule, da ihnen dies langfristig als einzig richtige Investition erscheint:

Als ich meine Mutter einmal fragte, warum wir alle drei [Geschwister] am Gymi waren und warum gar nie etwas anderes diskutiert wurde, sagte sie, dass sie bei uns allen halt ein grosses Potenzial gesehen hat. Von dem her fand sie es schon wichtig, dass wir dieses auch ausschöpften. (Veronika, 21 Jahre)

Durch den fördernden Einfluss der Eltern, insbesondere der Mutter, schrieb sich Veronika schliesslich an einer Universität ein und studierte Jurisprudenz. Aufgrund ihres ausgeprägten Interesses am Themenschwerpunkt Internationale Beziehungen und durch ihren Onkel, der bei einer Regierungsorganisation in diesem Bereich tätig ist, gelang es Veronika bereits auf Bachelorstufe, sich erfolgreich für ein Praktikum zu bewerben. Hier kann also auch der Onkel als förderndes Familienmitglied mit Vorbildfunktion bezeichnet werden.

Ein gegenteiliges Bild zeigt sich hingegen bei Katja. Im Interview mit Katja wird deutlich, dass die Familie sich nicht mit dem beruflichen Werdegang der Tochter identifizieren konnte und sie deshalb bei ihrer Berufsausübung auch nicht unterstützte:

Meine Mutter wollte immer, dass ich das KV mache und so in ihre Richtung gehe. (...) Aber jetzt ist auch dort die genau gleiche Barriere. Mein Vater ist eigentlich noch eher der, der sich dafür [Beruf] interessiert. (...). Aber sobald er dann fragt, was machst du denn genau? Was ist das für Forschung, wo du da betreibst beim Arbeiten? Dann ist immer die erste Frage: Ja, wer bezahlt das? Wer braucht das? (..). Also ja, so was halt. Wirklich fremd. (Katja, 26 Jahre)

Wie Veronika hat auch Katja ein Gymnasium absolviert.

Blockierend sind signifikante Andere zu bezeichnen, die den Frauen explizit von einer schulischen oder beruflichen Laufbahn abraten oder den Frauen bei der Wahl einer Ausbildung oder eines Berufes hinderlich erscheinen. Katja bezeichnet ihre Eltern, vor allem ihre Mutter, als blockierend und hindernd. Während Katjas Mutter die Einmündung in einen Dienstleistungsberuf (Sachbearbeiterin) als völlig ausreichende Qualifizierung für ihre Tochter erachtet, strebt Katja eine höhere Schulbildung im Tertiärbereich an. Katja deutet den blockierenden Einfluss der Mutter so, dass «ich halt ein deutlich höheres Bildungsniveau als sie [die Eltern] habe». Mit Katjas Studium in den Gesellschaftswissenschaften konnten die Eltern, wie sie sagt, nie etwas anfangen und Forschung in diesem Bereich erachten die Eltern als Ressourcenverschwendung. Die eingeschlagene akademische Karriere von Katja ist vorwiegend auf ihren Gymnasiallehrer zurückzuführen. Aufgrund ihrer guten Schulnoten und seiner ausdrücklichen Empfehlung für ein Studium fühlte sich Katja in ihrer Entscheidung, an die Uni zu gehen, bestärkt. Am Beispiel von Katja zeigt sich, dass zum gleichen biogra- 
phischen Zeitpunkt auch gegenteilige Einflüsse von verschiedenen Personen parallel wirken können. Begünstigt durch den Gymnasiallehrer erlangte Katja eine habituelle Sicherheit (Giddens, 1991; Schwiter, Wehner, Maihofer und Huber, 2011), welche es ihr ermöglichte, sich über den Einfluss der Eltern hinwegzusetzen.

Die Analyse der Interviews konnte einen weiteren Einfluss von Seiten der Eltern ausmachen. Am Beispiel von Nicole zeigt sich ein beratender Einfluss des Vaters. Nicoles Vater, der eine Berufsausbildung in der Elektrobranche absolvierte, in verschiedenen Bereichen gearbeitet hat und auf ein grosses Netzwerk zurückgreifen kann, empfiehlt seiner Tochter eine kaufmännische Lehre und versucht auf die Optionsvielfalt in diesem Bereich hinzuweisen.

Ja, ich denke sicher, mein Vater, also ich, ja, als ich nicht wusste, was ich lernen soll. Ich fand das damals mega schwierig. Dann haben wir halt darüber diskutiert und er meinte: Wenn du dir nicht so sicher bist, dann mach doch das $\mathrm{KV}$, dann bist du nachher sicher nicht verloren, ja, das ist so breit. (Nicole, 27 Jahre)

Als beratend sind also diejenigen signifikanten Anderen zu bezeichnen, welche insbesondere auf Möglichkeiten und die Optionsvielfalt von schulischen und beruflichen Verläufen und Weiterbildungen hingewiesen haben. Interessant ist hier insbesondere die Empfehlung des Vaters zur Wahl eines neutralen Berufes $(\mathrm{KV})^{3}$, der im Gegensatz zu vielen typischen Frauenberufen mehr Aufstiegsund Weiterbildungsmöglichkeiten mit sich bringt. Die Mutter bringt Nicole in Zusammenhang mit der Berufswahl nicht zur Sprache. Durch das soziale Kapital (Bourdieu, 1983) des Vaters konnte Nicole im Bereich Marketing ihre Lehre beginnen. Auch nach der Lehre arbeitete Nicole weiter in diesem Bereich. Sie bekam nach eigener Aussage von ihrem Vorgesetzten positive Rückmeldungen und fühlte sich deshalb beruflich am richtigen Platz. Dieses Beispiel zeigt, dass eine Kumulation von positiven Rückmeldungen für den weiteren Ausbildungsund Berufsverlauf entscheidend sein kann. Nicole konnte einerseits auf den beratenden Einfluss des Vaters zurückgreifen, andererseits aber auch auf einen fördernden Einfluss des Vorgesetzten.

Die vierte Gruppe der in dieser Studie identifizierten Einflüsse ist weder explizit fördernd noch beratend oder blockierend. Vielmehr ist sie als hinnehmend zu bezeichnen. Eine gewisse Gleichgültigkeit bezüglich der Berufswahl der Tochter ist an folgendem Zitat auszumachen: "Mein Vater hat einfach gesagt: Mach irgendetwas. Ihm war es nicht so wichtig, was ich mache." (Irina, 24 Jahre). Die berufliche Wahl oder der berufliche Verlauf wird hier weitgehend hingenommen. Irina erklärt sich das Verhalten des Vaters so, dass er aufgrund seiner griechischen Abstammung und dadurch, dass er selber in der Schweiz nie einer Erwerbstätigkeit nachgegangen ist, auch keine Empfehlungen abgeben konnte. Vielmehr 
sieht er die Rolle seiner Tochter als zukünftige Hausfrau, welche sich primär um die Hausarbeit und die Betreuung ihrer Kinder kümmert. Eine grössere Investition in Bildung erscheint für ihn deshalb eher unnötig. Irina wurde in der Schweiz geboren und hat ihre obligatorische Schulzeit in der Schweiz absolviert. Sie sieht ihre eigene berufliche Partizipation als notwendigen Schritt für ihre eigene Selbstständigkeit und Loslösung vom Elternhaus. Nach einer «Fehlentscheidung», wie Irina sagt, im Detailhandel ihre Lehre zu absolvieren, entschliesst sie sich schliesslich für ein KV mit Berufsmaturitätsschule und studiert derzeit an einer Fachhochschule Wirtschaft.

Generell wird in den Interviews deutlich, dass die Einflüsse der einzelnen Familienmitglieder oft situationsabhängig sind und sich im zeitlichen Verlauf verändern können. Oftmals sind die Einflüsse von der schulischen oder beruflichen Situation der jungen Frauen abhängig, wechseln deshalb oft und bewegen sich zwischen mehreren der oben genannten Einflüsse. Teilweise empfinden die jungen Frauen Einflüsse auch anders, als sie beispielsweise von den Eltern verstanden werden. Gut gemeinte Ratschläge oder Angebote für die Finanzierung von Schulen und Weiterbildungen, die von den Eltern als fördernde Massnahmen angesehen werden, können bei den Frauen subjektiv als hindernd oder sogar blockierend verstanden werden. Bei folgendem Beispiel zeigt sich, dass die Laisser-faire-Erziehung der Eltern, die als fördernde Massnahme gedacht war, von Laura eher als blockierend empfunden wurde:

Sie hatten einfach sehr das Vertrauen, ja, es kommt dann schon gut irgendwie. Früher hätte ich es mir manchmal mehr gewünscht, wenn sie mir, ja nicht eine Richtung vorgeben, aber... Ja, ich war schulisch nie schlecht, bin aber einfach immer im Minimum geblieben von meinem Fassungsvermögen. Ich glaube, es gibt dann schon eine Zeit, in der die Eltern dann auch helfen können, dass man etwas dranbleibt. Gerade in der Pubertät, von der einen Seite zur anderen. Ja, teilweise waren wir vielleicht fast etwas zu frei. Wir hatten dann gar keinen Halt mehr. Dann schleift das so hin und her und dann muss man das dann halt selber merken. (Laura, 25 Jahre)

Laura begründet ihre berufliche Orientierungslosigkeit einerseits aufgrund einer Langzeiterwerbslosigkeit beider Elternteile, einer zu grossen räumlichen Trennung zu den Eltern (die Eltern leben im Ausland), aber auch aufgrund einer generellen "Haltlosigkeit». Nach dem erfolgreichen KV-Abschluss fühlte sich Laura in der Firma zunehmend «unpassend». Ihre Leistungen, sagt sie, seien deutlich hinter dem zurückgeblieben, was sie eigentlich konnte. Zudem gelang es ihr nicht, sich selber für eine Weiterbildung zu motivieren und gab schliesslich ihren Beruf auf. Laura ist seit einigen Jahren Hausfrau und Mutter.

Im vorliegenden Datenmaterial zeigt sich die Tendenz, dass das berufliche und intellektuelle Anforderungsniveau der Mutter im Laufe der Zeit überschritten 
wird oder zumindest gleich bleibt. Keine der interviewten jungen Frauen befindet sich unter dem Anforderungsniveau der Mutter, jedoch befinden sich fast alle unter dem beruflichen Anforderungsniveau des Vaters. Väter werden deshalb oft bei der Entscheidungsfindung bezüglich weiterführenden Aus- und Weiterbildungen, insbesondere auch in Zusammenhang mit der Finanzierung eines zusätzlichen Bildungszertifikats, eingebunden und stehen den jungen Frauen in dieser Hinsicht beratend und fördernd zur Seite. Das folgende Zitat verdeutlicht, dass Simone versucht ist, das Anforderungsniveau der Mutter zu überschreiten, in dem sie die Maturitätsprüfung macht. Simone gibt an, dass ihr erster Berufswunsch Ärztin war. Motiviert durch ihren Vater, der Zahnarzt ist, versuchte sie, sich seinem Bildungsniveau anzugleichen. Dies gelang ihr jedoch nicht - sie bestand die Gymnasialprüfung nicht. Simone absolvierte schliesslich eine Lehre als Dentalassistentin. Seit der Geburt ihrer Tochter vor einigen Monaten ist Simone nicht mehr erwerbstätig.

Ja eben, meine Mutter war auch Dentalassistentin. Und es war schon so damals, als ich die Kantiprüfung nicht bestanden hatte und mich entscheiden musste was ich machen soll: ob Dentalassistentin oder Arztgehilfin. Dann habe ich gedacht, nein, ich will nicht in die gleiche Richtung gehen wie Vater und Mutter und stelle mich jetzt extra dagegen und probiere es mit der Arztgehilfin. Ich habe dann eben leider nichts gefunden und ja. Ich habe dann als Dentalassistentin mein neues Glück versucht und habe dann Gott sei Dank etwas gefunden. Dann habe ich einfach gedacht, jetzt machst du die drei Jahre und kannst dann immer noch schauen, was du dann machst. (Simone, 26 Jahre)

In Bezug auf Ausbildungs- und Berufsverläufe, aber auch Berufsfindungsprozesse argumentieren Familienmitglieder und FreundInnen zumeist stark in Richtung ihres eigenen beruflichen Werdegangs. Ihre eigene berufliche Partizipation und ihre Erfahrungen dienen oft als Ausgangslage für die berufliche oder schulische Weiterentwicklung der interviewten jungen Frauen. Im zeitlichen Verlauf wird sichtbar, dass sich der Einfluss der Eltern mit zunehmender schulischer oder beruflicher Partizipation verringert, dafür der Einfluss der FreundInnen an Bedeutung gewinnt.

\section{FreundInnen}

Die Gruppe der FreundInnen beinhaltet sowohl enge FreundInnen, Bekannte, MitschülerInnen, KommilitonInnen, als auch BerufskollegInnen. In den Interviews zeigt sich insbesondere in Bezug auf den ausgeübten Beruf und mit zunehmendem Alter eine Tendenz zur Überlappung der Einflüsse von Familie und FreundInnen. Die Überlappung mehrerer Gruppen wird im folgenden Beispiel deutlich:

Das ist, ja das ist schon ein Thema, weil sie immer gesagt haben: Was machst du mit dem? Weil sie den Praxisbezug gesucht haben und schon die Matur 
etwas Spezielles war für sie. Dann war halt immer die Frage: Was willst du mit dem nachher machen? Oder sogar jetzt. Also meine Mutter weiss nicht, was ich genau mache. Mein Vater auch nicht. Und wenn sie dann fragen, was arbeitest du denn mal? Ja, sie können wirklich nichts damit anfangen. Das ist extrem. Wir können uns auf dieser Schiene fast nicht unterhalten. Das ist für sie so etwas Weltfremdes, irgendwie. In meinem Freundeskreis ist das eigentlich kein Thema, weil die fast alle studieren. (Katja, 26 Jahre)

Dieses Zitat illustriert, dass insbesondere zu Beginn der Ausbildung beziehungsweise Berufsfindung die Eltern versucht sind, richtungsweisende Ratschläge in Bezug auf den Ausbildungs- oder Berufsverlauf zu geben. Mit zunehmendem Alter und zunehmender Einbindung in die Peergroup zeigt sich hingegen ein schwächerer Einfluss der Eltern, dafür ein zunehmender Einfluss der FreundInnen und KollegInnen, die sich zumeist in einer ähnlichen schulischen oder beruflichen Situation befinden. Der Einfluss muss jedoch nicht zwingend, wie bei diesem Fall, fördernd sein. Das folgende Zitat zeigt einen eher blockierenden Einfluss der Freundinnen: «Aber eben, die Kolleginnen haben oft gesagt, wie kannst du das, so etwas machen. Computer und all das Zeugs wäre besser, dann bekommst du auch mehr Lohn.» (Simone, 26 Jahre). Es macht deutlich, dass sich die Kolleginnen gegen den Berufswunsch von Simone aussprechen. Während sich Simone für eine Ausbildung als Arztgehilfin oder Dentalassistentin interessiert, sprechen sich ihre Freundinnen, die entweder im Gymnasium oder in einer kaufmännische Lehre sind, gegen ihren Wunsch aus. Die Freundinnen hat Simone zu diesem Zeitpunkt eher als blockierend verstanden. Nichtsdestotrotz hat sich Simone für eine Ausbildung als Dentalassistentin entschieden. Während für Simones Freundinnen finanzielle Aspekte bei der Berufsentscheidung eine grosse Rolle spielten, stand der Lohn für Simone zu diesem Zeitpunkt nicht im Vordergrund.

Eine Einflussverlagerung von den Eltern zu den FreundInnen oder KollegInnen geht mit steigendem Alter und eigener schulischer und beruflicher Partizipation einher. Mit zunehmender Einbindung ins Berufsleben oder weiterführenden Schulen nimmt der Einfluss der LehrerInnen, LehrmeisterInnen und Vorgesetzten insbesondere gegenüber den Eltern zu. Die Hauptgruppe LehrerInnen, LehrmeisterInnen und Vorgesetzte wird in folgendem Abschnitt näher beleuchtet.

\section{LehrerInnen, LehrmeisterInnen und Vorgesetzte}

Als weitere Gruppe signifikanter Anderer konnte die der LehrerInnen, LehrmeisterInnen, aber auch Vorgesetzten identifiziert werden. Besonders zu Ende der obligatorischen Schulzeit nehmen die interviewten jungen Frauen einen fördernden Einfluss von LehrerInnen, insbesondere in Bezug auf eine weitere schulische Bildung, wahr. Auch LehrmeisterInnen weisen gegen Ende der Berufslehre auf berufliche Weiterbildungen hin und können somit sowohl als fördernd als auch als beratend bezeichnet werden. 
Während der Adoleszenz gelten LehrerInnen aller Art oft als Vorbilder für die jungen Frauen. Aber auch mit zunehmendem Alter und konkreteren beruflichen Vorstellungen zeigt sich, welch starken Vorbildcharakter LehrerInnen ausüben können:

Ich wollte dann ja in der 4. Klasse raus, wegen meinem Tierärztin-Wunsch. Ich kannte eben eine Kantilehrerin und sie hatte halt gut von der Kanti gesprochen. Mit der Steinerschule war das eben, ja gut, man konnte schon die Matur machen, aber es war alles noch nicht ganz so klar mit der Anerkennung und allem. Deshalb konnte sie mich auch sehr für das begeistern. (Laura, 25 Jahre)

Laura erzählt vom starken Vorbildcharakter ihrer Gymnasiallehrerin. Motiviert durch diese Lehrerin verliess Laura die Steinerschule und verbrachte ihre restliche Primarzeit an einer staatlichen Schule.

Dann bin ich dann raus, in die Primarschule, dann in die 1. Sek, dann ist mir der Laden so runter, dass ich effektiv wieder zurück in die Steinerschule gegangen bin. (Laura, 25 Jahre)

In der Sekundarschule zeigte sich für Laura, dass sie sich nicht zurechtfinden konnte, ihre Vorstellungen von einer Sekundarschule nicht mit dem übereinstimmten, was sie tatsächlich erlebte, und sie sich zunehmend als Aussenseiterin fühlte. Ihre habituelle Sicherheit und ihre Motivation, Tierärztin zu werden, gingen verloren. Laura bezeichnet ihre Gymnasiallehrerin auf der einen Seite als fördernd in Bezug auf ihren Wunschberuf, gleichzeitig aber auch als blockierend, weil sie in ihr "falsche Vorstellungen geweckt» hätte. Ein wiederholter Schulwechsel führte nach eigenem Bekunden zu einer zunehmenden Orientierungslosigkeit auf ihren weiteren Ausbildungs- und Berufsverlauf.

LehrmeisterInnen, aber auch Vorgesetzte werden von den jungen Frauen heterogen dargestellt. Einerseits werden sie als positiv und fördernd beschrieben:

Es war so, dass mir das mein Chef vorgeschlagen hat. Weil wir ein Gespräch hatten und er meinte, dass er sehr zufrieden sei mit mir. Und dann haben wir besprochen, wenn es Möglichkeiten gibt, in welche Richtung will ich? Und dann habe ich gesagt ich möchte lieber in Richtung Marketing als in Verkauf. Verkaufsaussendienst oder so kommt nicht in Frage. Dann meinte er, ob ich nicht eine Schule machen will. Dann könnte er auch sagen: Sie ist dafür ausgebildet und befähigt. Was auch immer. Und dann habe ich mir das einmal angeschaut. Und ihn dann auch gefragt: Wie sieht es denn mit der Finanzierung aus? Er meinte dann: Ja, das ist kein Problem, das können wir bezahlen. (Nicole, 27 Jahre)

Dieses Zitat macht den beratenden Einfluss des Vorgesetzten deutlich. Nicole fühlte sich in dieser Firma aufgehoben, erlangt durch positive Rückmeldungen ihres Vorgesetzten zunehmend habituelle Sicherheit und investiert dadurch in weiteres institutionalisiertes Kapital. 
Im Gegensatz dazu zeigt sich am Zitat von Irina, wie sehr sie ihre Vorgesetzte als Belastung empfindet:

Das war so ein Biest! Ich konnte schon gar nicht mehr schlafen, hatte richtig Angst und Panik, überhaupt da hinzugehen. Dann habe ich gekündigt. (Irina, 24 Jahre)

Hier zeigt sich ein blockierender Einfluss von Seiten der Vorgesetzten, welcher Irina schliesslich zu einer Kündigung ihres Lehrverhältnisses im Detailhandel bewegte. Sie entschied sich daraufhin für eine KV-Lehre bei einer Bank.

Werden die einzelnen bisher diskutierten Gruppen im zeitlichen Verlauf angesehen, zeigt sich, dass der Einfluss der Familie und FreundInnen im früheren Alter besonders stark ist. Mit zunehmendem Alter verschiebt sich der Einfluss jedoch in Richtung LehrerInnen, LehrmeisterInnen und Vorgesetzte. Ebenfalls gewinnt die heterogene Einflussgruppe mit zunehmendem Alter an Bedeutung, wie der folgende Abschnitt zeigt.

\section{Heterogene Einflussgruppe}

Die letzte durch die qualitative Inhaltsanalyse identifizierte Gruppe ist als heterogen $\mathrm{zu}$ bezeichnen. In dieser Gruppe befinden sind beispielsweise Personen, die im Zusammenhang mit professionellen Settings genannt werden. Diese Personen kommen insbesondere in Bezug auf Wunschberufe zur Sprache, da sie oftmals als berufliche Vorbilder gelten:

Ich denke, es war wegen Lonely Planet. Dort arbeiten alles Junge, die bezahlt werden fürs Reisen und dann einen Reisebericht schreiben. So etwas wäre wirklich gut. Reiseberichte. Das wäre echt cool. (Sybille, 25 Jahre)

Obwohl diese ReiseberichterstatterInnen für Sybille unbekannte Personen sind, gelten diese für sie als berufliche Vorbilder. Nach einem erfolgreichen Abschluss als Damenschneiderin hat sich Sybille beruflich entsprechend umorientiert. Sie ist nun in der Reisebranche tätig. Ihre Arbeit am Flughafen empfindet sie als optimale Kombination, um Hobby und Beruf zu verbinden. Sie bezeichnet die ReiseberichterstatterInnen sowohl als fördernd als auch als beratend für ihren eigenen Berufsweg. Durch diese Reiseberichte hätte sie gemerkt, wohin sich ihre eigenen beruflichen Ziele entwickeln. Derzeit lebt Sybille in Thailand und bietet Führungen für Reisegruppen an.

In dieser heterogenen Gruppe ist beispielsweise auch eine Psychologin genannt worden. Laura bezeichnet ihre Psychologin als persönliche Stütze während einer Phase der Orientierungslosigkeit und Selbstfindung:

Sie hat, also meine Psychologin, sie war natürlich schon sehr wichtig. Mit ihr konnte ich auch über alles reden. Es ging zwar nicht konkret um den Beruf, aber einfach so als Anlaufstelle. (Laura, 25 Jahre) 
Lauras Psychologin stand zwar, im Gegensatz zu den Reiseberichterstattern von Sybille, in persönlichem Kontakt zu ihr, jedoch war sie nur für eine kurze Dauer bedeutend für Laura. Sie bezeichnet ihre Psychologin einerseits als fördernd in Bezug auf einen Ausweg aus der Orientierungslosigkeit, andererseits als beratend und als Anlaufstelle.

Oft wird der Einfluss der heterogenen Gruppe als fördernd und beratend bezeichnet, da diese Personen zumeist selektiv in bestimmten zeitlich begrenzten Situationen relevant war. Der konkrete Einfluss der heterogenen Gruppe kann im zeitlichen Verlauf jedoch nicht näher beurteilt werden, da diese Gruppe sehr diffus ist. Auch handelt es sich durchwegs um Personen, die nur für eine kurze Zeitdauer von Bedeutung sind, schnell wechseln können oder sogar austauschbar sind. Zusätzlich zeigt sich, dass die genannten Personen oder Personengruppen nicht in persönlichem Kontakt zu den interviewten Frauen stehen müssen.

\section{Gendering}

Insbesondere bei den Eltern zeigen sich geschlechtsspezifische Besonderheiten in ihrem Verhalten gegenüber ihren Töchtern. In den geführten Interviews wird deutlich, dass Mütter sich oftmals für die schulische und berufliche Weiterentwicklung ihrer Töchter verantwortlich fühlen und in dieser Hinsicht emotionell-fördernde Hilfestellungen bieten wollen. Geht es hingegen um schulische oder berufliche Finanzierungen, werden zumeist die Väter angesprochen. Das folgende Zitat veranschaulicht die finanzielle Hilfestellung des Vaters:

Da fanden alle in der Familie: Es wird ja doch noch etwas aus diesem Kind. Ja, da waren alle zuversichtlich. Ausser eben das Finanzielle. Aber mein Vater hat gesagt, es sei easy. Er finanziere meiner Schwester ja auch das Studium. Deshalb sei es auch ganz ok, wenn ich eine zweite Ausbildung mache. Das sei völlig legitim. (Dina, 26 Jahre)

Inwieweit für die Töchter eine weitere Investition in Bildung nun attraktiv erscheint, zeigt sich nicht nur anhand der Einflussnahme der Eltern, sondern auch in Abhängigkeit ihrer schulischen oder beruflichen Orientierung und weiteren signifikanten Anderen wie beispielsweise FreundInnen, LehrerInnen und Vorgesetzte. Die Analyse macht auch deutlich, dass sowohl die Einflussnahme, aber auch die Orientierungshilfe der Eltern meist entlang geschlechtstypischer Argumentationslinien verlaufen. Wird davon ausgegangen, dass Mütter aus früheren Generationen weniger berufliche Optionen hatten und ihren Töchtern aufgrund ihrer eigenen beruflichen Partizipation Empfehlungen abgeben, führt dies oft dazu, dass die Töchter von bestimmten beruflichen Wünschen vorzeitig abkommen und so nie oder zumindest weniger in den Einfluss von LehrerInnen, LehrmeisterInnen und Vorgesetzten gelangen. Der Einfluss der LehrerInnen und LehrmeisterInnen, aber auch Vorgesetzten hat zumeist einen positiven Einfluss auf die weiterführende schulische und berufliche (Weiter-)Bildung der interviewten jungen Frauen. 
In den Interviews zeigte sich, dass insbesondere Lehrerinnen im frühen Alter als berufliches Vorbild für die jungen Frauen gelten. Vor allem in Bezug auf den Wunschberuf im Kindesalter wird in unserer Stichprobe eine auffällig starke Orientierung an weiblichen Lehrpersonen (Kindergartenlehrerinnen, Primarlehrerinnen, aber auch z. B. einer Reitlehrerin) deutlich.

Mir hat es eben im Kindsgi und in der Primar so gut gefallen, die Lehrerinnen waren eben auch so toll dort. (Stefanie, 26 Jahre)

Ein in der Schule ausgeprägter institutionalisierter Genderismus, gekoppelt mit Vorstellungen von mütterlicher Versorgung (Goffman, 2001, S. 135) trägt demnach zu einem stark geschlechtstypischen Berufswunsch bei jungen Frauen bei. Dies kann bereits als geschlechtsspezifische Weichenstellung verstanden werden. Vorstellungen von Weiblichkeit und weiblicher beruflicher Partizipation werden unseren Befunden zufolge einerseits von Seiten der Mutter, andererseits aber auch von Seiten der Lehrerinnen reproduziert. Da mit steigendem Alter und zunehmender schulischer wie beruflicher Partizipation der Einfluss der Mütter und der Lehrerinnen weitgehend abnimmt und dafür der Einfluss von FreundInnen zunimmt, zeigt sich schliesslich oft die Wahl eines zumindest geschlechtsneutralen Berufs (meist kaufmännische Ausbildungen). Generell wird aber deutlich, dass von den jungen Frauen oftmals ein geschlechtstypischer Beruf ergriffen wird. Geschlechtsuntypische Berufe werden von Seiten der jungen Frauen selten in Betracht gezogen. Dina bildet in diesem Datenmaterial die Ausnahme. Das folgende Zitat illustriert den blockierenden Einfluss des Freundes und schliesslich auch der Eltern auf den geschlechtsuntypischen Berufswunsch von Dina:

Ich hatte einen Freund, als ich 16 war, bis 18. Er war Schreiner und ich wollte da ja auch reinschauen, was ich auch gemacht habe. Dort hat er, also er fand nicht, dass das etwas für mich wäre, das sei eine Männersache. Und von dem her hat er mich halt beeinflusst, weil ich dann dachte: Und ob ich das kann! Aber ich habe halt wirklich keine Lehrstelle gefunden und ich war damals halt noch extrem, also ich war noch ein extremes «Finöggeli». Dann fanden sie [Eltern und Freund] halt alle, hmm, auch wegen dem Tragen und so das schwer Schleppen sei nicht gesund für mich. Dann habe ich das halt nicht gemacht. (Dina, 26 Jahre)

Für Dina stellt die mögliche Ausübung eines geschlechtsuntypischen Berufs "nicht zwangsmässig die (biologische und soziale) Geschlechtsidentität in Frage» (Trautner, 2006, S. 105). Hingegen nehmen Dianas Freund und ihre Eltern den geäusserten Berufswunsch als Bruch wahr, als unakzeptablen Berufswunsch für ein Mädchen. Der vergeschlechtlichte Einfluss der Familie verläuft oft entlang der Argumentationen der selber erlebten beruflichen Partizipation der Mutter und endet damit in einem geschlechtstypischen Beruf der Tochter. Da die eigene berufliche Partizipation der Mütter mehrheitlich in geschlechtstypischen Bahnen verlief, transferiert sich diese Neigung auch auf ihre Töchter. Bei der 
Wahl eines geschlechtstypischen Berufs wird oft davon ausgegangen, dass sich diese für das jeweilige Geschlecht einerseits besonders gut eignen, andererseits aber auch, dass damit dem von Bourdieu (1983) beschriebenen geschlechtsspezifischen Ideal gerecht werden kann. Die geschlechtsspezifische Sozialisation führt also dazu, dass junge Frauen oft frauentypische Berufe "wählen», weil sie davon ausgehen, dort besonders passend zu sein. Mit steigendem Alter und eigener beruflichen Partizipation zeigt sich jedoch, dass es Jugendlichen zunehmend leichter fällt, Geschlechtergrenzen zu überschreiten und zumindest einen neutralen Beruf zu ergreifen. Insbesondere Frauen scheint es leichter zu fallen, Geschlechtergrenzen zu überwinden. Dies kann mit oftmals besseren Rahmenbedingungen (z.B. höheren Löhnen, bessere Aufstiegsmöglichkeiten etc.) in männertypischen Berufen begründet werden (Hupka-Brunner, Huber \& Bergmann, in Bearbeitung). Es zeigen sich aber generell nur wenige junge Frauen bereit, in einen geschlechtsuntypischen Beruf einzumünden. Viele fühlen sich den Anforderungen eines typisch männlichen Berufes nicht gewachsen, da diese oftmals mit körperlicher Anstrengung verbunden sind (Cornelissen, 2009). Zudem ergeben sich insbesondere in der Pubertät oft Konflikte mit der eigenen Geschlechtsidentität, der Darstellung von Geschlecht, aber auch einem Druck von Seiten der Peergroup, sich entsprechend geschlechtsspezifisch zu verhalten.

Insgesamt wird im Datenmaterial deutlich, dass wichtiger ist, wie der Einfluss auf die jungen Frauen erfolgt, weniger jedoch, wer Einfluss ausübt. Auch ist entscheidend, wann der Einfluss genau erfolgt: Im frühen Kindesalter ist der Einfluss der signifikanten Anderen stärker als mit zunehmendem Alter und zunehmender beruflicher Partizipation. Ist der Einfluss der Eltern, LehrerInnen und LehrmeisterInnen fördernd oder beratend, zeigt sich einerseits eine höhere Tendenz, eine weiterführende Schule zu besuchen, andererseits aber auch zur Wahl eines neutralen Berufs. Ist der Einfluss hingegen hinnehmend oder sogar blockierend, wählen die interviewten jungen Frauen eher einen geschlechtstypischen Beruf, zeigen aber doch in dieser nicht-repräsentativen Stichprobe eine auffällige Tendenz, später weiterführende Bildungszertifikate zu erwerben.

\section{Faz it}

Zusammenfassend zeigt sich im Datenmaterial, dass von den interviewten jungen Frauen insgesamt vier Gruppen von signifikanten Anderen identifiziert werden konnten: Eltern, LehrerInnen/LehrmeisterInnen/Vorgesetzte, FreundInnen und eine heterogene Einflussgruppe. In Bezug auf den Ausbildungs- und Berufsverlauf konnten die jungen Frauen zumeist klar benennen, wer für sie bedeutsam war und wie sie deren Einflüsse wahrgenommen haben. Die Einflüsse dieser Personen werden als hinnehmend, fördernd, blockierend und beratend beschrieben. Die vier Arten von Einflüssen gelten als Idealtypen 
- Mischformen sind oft vorzufinden. Der konkrete Einfluss dieser signifikanten Anderen verändert sich im Laufe des Alters und beruflicher Partizipation der jungen Frauen. Die Wirkung entfaltet sich auch oft auf mehreren Ebenen (individuell, institutionell, geschlechternormativ) gleichzeitig und teilweise in entgegengesetzter Richtung. Während sich beispielsweise Eltern gegen eine (untypische) Ausbildung der Tochter aussprechen und somit als blockierend bezeichnet werden können, fördern LehrerInnen diese Frauen in Richtung weiterführende Schule. Der Einfluss der Eltern ist insbesondere in jungen Jahren sehr ausgeprägt. Auch zeigen sich hier bereits in sehr jungen Jahren die Auswirkungen einer geschlechtstypischen Sozialisation durch die Mütter. Dies wird von allem anhand der genannten Wunschberufe deutlich. Die Wunschberufe orientieren sich auffallend oft an weiblichen Lehrpersonen oder anderen weiblich geprägten Berufe, welche mit Vorstellungen von mütterlicher Versorgung in Zusammenhang gebracht werden. Eine Vorbildfunktion und Idealisierung insbesondere von Kindergarten- und Primarlehrerinnen verstärken diese Geschlechtersegregation zusätzlich. Insbesondere Mütter neigen dazu, ihren Töchtern aufgrund ihrer eigenen beruflichen Partizipation geschlechtstypische Ausbildungen und Berufe zu empfehlen. Dieses Resultat könnte zum Schluss führen, dass insbesondere Mütter geschlechtstypische Verläufe fördern. Ergibt sich im Laufe des Ausbildungsund Berufsverlaufes ein stärkerer Bezug zu weiteren signifikanten Anderen wie LehrerInnen, LehrmeisterInnen oder Vorgesetzte, wird eine weniger ausgeprägte geschlechtsspezifische Sozialisation deutlich. Geschlechtsspezifische Zuweisungen zeigen sich im späteren Verlauf weniger personenabhängig. Viel eher werden sie von Institutionen vorgegeben und prägen so den weiteren Ausbildungs- und Berufsverlauf von jungen Frauen. Je früher die jungen Frauen demnach in den Einfluss weiterer signifikanter Anderer kommen, desto grösser ist die Wahrscheinlichkeit, dass neutrale oder sogar geschlechtsuntypische Ausbildungs- und Berufsverläufe gewählt werden.

Das Datenmaterial macht weiter deutlich, dass eine Überschreitung von geschlechtstypischen Grenzen und das Erreichen einer Geschlechtsparität in vielen Berufen nur unter bestimmten Voraussetzungen erreicht werden kann. Als entscheidend für eine potenzielle Überwindung erachten wir zum einen den Einfluss signifikanter Anderer: Lehnen die signifikanten Anderen, insbesondere die Eltern, die Wahl eines geschlechtsuntypischen Berufes ab, erscheint eine Einmündung in einen solchen als schwierig. Ein fördernder und beratender Einfluss der signifikanten Anderen kann jedoch für die Einmündung in einen zumindest neutralen oder geschlechtsuntypischen Berufes förderlich sein.

Weiter empfiehlt es sich zum anderen, die berufliche Orientierung der jungen Frauen bereits früh in der Schule zu thematisieren, da die jungen Frauen aufgrund ihrer geschlechtsspezifischen Sozialisation oft davon ausgehen, dass sie sich in einem frauentypischen Beruf besonders passend fühlen werden. Von 
Seiten der LehrerInnen und der Berufsberatung könnte insbesondere auf die geschlechtsuntypische Berufswahl aufmerksam gemacht werden.

Auf der theoretischen Ebene erscheinen sowohl Goffmans institutionalisierter Genderismus als auch Bourdieus Geschlechterhabitus als wenig aussagekräftig, um die Einflïsse der signifikanten Anderen genau zu beleuchten. Fruchtbar erscheinen diese Theorien jedoch in Bezug auf das gendering. Das Zusammenwirken von vergeschlechtlichten Wahrnehmungs-, Denk- und Handlungsschemata in den langfristigen Prozessen von Ausbildungs- und Berufsverläufen kann durch diese Theorien hervorragend abgedeckt werden.

\section{Anmerkungen}

1 Fellow der African Doctoral Academy and CREST, Stellenbosch, South Africa

2 Von einem "Frauenberuf" bzw. "Männerberuf" wird dann gesprochen, wenn im entsprechenden Beruf der Frauenanteil bzw. Männeranteil mehr als 70\% beträgt.

3 KV: Abkürzung für kaufmännischer Verein, bezeichnet wird damit in der Schweiz aber eine kaufmännische Ausbildung

\section{Literaturverzeichnis}

Baumgartner, A. D. \& Fux, B. (2004). Und sie bewegen sich doch nicht: die Männer. Zur geschlechtsspezifischen Verteilung der Erwerbsarbeit in Familien. In E. Zimmermann, R. Tillmann (Hrsg.), Vivre en Suisse 1999-2000. Leben in der Schweiz 1999-2000. Une année dans la vie des ménages et familles en Suisse. Ein Jahr im Leben der Schweizer Familien und Haushalte. Bern: Verlag Peter Lang.

Becker, R. (Hrsg.). (2008). Bildung als Privileg. Erklärungen und Befunde zu den Ursachen der Bildungsungleichheit. Wiesbaden: VS Verlag für Sozialwissenschaften.

Bergman, M. M., Hupka-Brunner, S., Keller, A. \& Meyer, T. (2011). Transitionen im Jugendalter: Ergebnisse der Schweizer Längsschnittstudie TREE. Zürich: Seismo.

Bergman, M. M., Hupka-Brunner, S., Keller, A., Meyer, T. \& Samuel, R. (2012). BildungArbeit-Erwachsenwerden. Wiesbaden: VS Verlag für Sozialwissenschaften.

Borkowski, A. (2000). Frauen und Männer in der Berufsbildung der Schweiz. Schweizerische Zeitschrift für Bildungswissenschaften, 22 (2), 279-293.

Born, C. \& Krüger, H. (2001). Individualisierung und Verflechtung. Geschlecht und Generation im deutschen Lebenslaufregime. Weinheim, München: Beltz Juventa.

Bourdieu, P. \& Passerson, J.-C. (1971). Die Illusion der Chancengleichheit: Untersuchungen zur Soziologie des Bildungswesens am Beispiel Frankreichs. Stuttgart: Klett.

Bourdieu, P. (1983). Ökonomisches Kapital, kulturelles Kapital, soziales Kapital. In R. Kreckel (Hrsg.), Soziale Ungleichheiten (S.183-198). Göttingen: Soziale Welt, Sonderband 2.

Bourdieu, P. (2005). Die männliche Herrschaft. Frankfurt/M: Suhrkamp.

Buchmann, M. \& Kriesi, I. (2009). Escaping the Gender Trap. Young Women's Transition into Non-Traditional Occupations. In I. Schoon \& R. Silbereisen (Hrsg.), Transition from School to Work. Cambridge and New York: Cambridge University Press.

Charles, M. (2005). Entwicklung der beruflichen Segregation nach Geschlecht und nach Staatsangehörigkeit in der Schweiz, 1970-2000. Neuchâtel: BfS.

Cornelissen, W. (2009). Die Relevanz von Geschlechterstereotypen für Berufswahlentscheidungen - eine Herausforderung für die Gleichstellungspolitik. In S. Baer u.a. (Hrsg.), Schubladen, Schablonen, Schema F-Stereotype als Herausforderung für Gleichstellungspolitik (S. 67-88). Bielefeld: Kleine Verlag. 
Cornelissen, W. \& Gille, M. (2005). Lebenswünsche junger Menschen und die Bedeutung geschlechterstereotyper Muster. Zeitschrift für Frauenforschung und Geschlechterstudien, 23 (4), 52-67.

Faltermeier, J. (2001). Verwirkte Elternschaft? Fremdunterbringung - Herkunftseltern - neue Handlungsansätze. Münster: Votum Verlag.

Giddens, A. (1991). Modernity and Self-Identity: Self and Society in the Late Modern Age. Cambridge: Polity.

Goffman, E. (2001). Interaktion und Geschlecht. Frankfurt am Main/New York: Campus Verlag.

Gottschall, K. (2009). Arbeitsmärkte und Geschlechterungleichheit - Forschungstraditionen und internationaler Vergleich. In B. Aulenbacher. \& A. Wetterer (Hrsg.), Arbeit. Perspektiven und Diagnosen der Geschlechterforschung (S. 120-137). Münster: edition sigma.

Green, J., Willis, K., Hughes, E., Small, R., Welch, N., Gibbs, L. \& Daly, J. (2007). Generating best evidence from qualitative research: the role of data analysis. Australian and New Zealand Journal of Public Health. 31 (6), 545-550.

Hupka-Brunner, S., Huber, E. \& Bergman, M. M. (in Bearbeitung). Typische und untypische Ausbildungs- und Berufsverläufe von Jugendlichen und jungen Erwachsenen.

Imdorf, C. (2004). Geschlechtsspezifische Selektion bei der Ausbildungsplatzvergabe. In Schweizerische Konferenz der Gleichstellungsbeauftragten, SKGB (Hrsg.), Achtung Gender: Ausbildungsverhalten von Mädchen und jungen Frauen. Trends und Tipps. Zürich: SVB.

Imdorf, C., Granato, M., Moreau, G., Waardenburg, G. \& Bergman, M. M. (2010). Sociology of Vocational Education and Training in Switzerland, France and Germany. Schweizerische Zeitschrift für Soziologie, 36 (1), 5-186.

Jäger, U., König, T. \& Maihofer, A. (2011). Pierre Bourdieu: Die Theorie männlicher Herrschaft als Schlussstein seiner Gesellschaftstheorie. In H. Kahlert \& C. Weinbach (Hrsg.), Zeitgenössische Gesellschaftstheorien und Genderforschung. Einladung zum Dialog. Opladen: Westdeutscher Verlag.

Kalthoff, H. (2004). Schule als Performanz. Anmerkungen zum Verhältnis von neuer Bildungsforschung und der Soziologie Pierre Bourdieus. In S. Engler \& B. Krais (Hrsg.), Das kulturelle Kapital und die Macht der Klassenstrukturen (S. 115-140). München: Juventa.

Krais, B. \& Gebrauer, G. (2002). Habitus. Bielefeld: transcript Verlag.

Leemann, R. J. \& Keck, A. (2005). Der Übergang von der Ausbildung in den Beruf. Bedeutung von Qualifikation, Generation und Geschlecht. Neuchâtel: Bundesamt für Statistik.

Levy, R. (1977). Der Lebenslauf als Statusbiografie. Die weibliche Normalbiografie in makrosoziologischer Perspektive. Stuttgart: Ferdinand Enke Verlag.

Maihofer, A., Bergman, M. M., Huber, E., Hupka-Brunner, S., Kanji, S., Samuel, R., Schwiter, K. \& Wehner, N. (2012). Kontinuität und Wandel von Geschlechterungleichheiten in Ausbildungs- und Berufsverläufen junger Erwachsener in der Schweiz. Zwischenbericht zum Forschungsprojekt zu Handen des Schweizerischen Nationalfonds.

Maihofer, A. (2004). Geschlecht als soziale Konstruktion - eine Zwischenbetrachtung. In U. Helduser u.a. (Hrsg.), Under Construction? Konstruktivistische Perspektiven in feministischer Theorie und Forschungspraxis (S. 33-43). Frankfurt/M., New York: Campus.

Maihofer, A. (2002). Geschlecht und Sozialisation. Erwägen Wissen Ethik, 13 (1), 13-26.

Ostendorf, H. (2005). Steuerung des Geschlechterverhältnisses durch eine politische Institution. Die Mädchenpolitik der Berufsberatung. Opladen: Verlag Barbara Budrich.

Schwiter, K., Wehner, N., Maihofer, A. \& Huber, E. (2011). Zur Hartnäckigkeit geschlechtsspezifischer Ausbildungs- und Berufsverläufe - Überlegungen zu einer empirischen Untersuchung. Femina Politica, 14 (2), 20-32.

Strub, S., Hüttner, E. \& Guggisberg, J. (2005). Arbeitsteilung in Paarhaushalten. Aufteilung von bezahlter und unbezahlter Arbeit in der Schweiz. Neuchâtel: Bundesamt für Statistik (aus Eidg. Volkszählung 2000. Büro für arbeits- und sozialpolitische Studien BASS, Bern). 
Trautner, H.-M. (2006). Sozialisation und Geschlecht. Die entwicklungspsychologische Perspektive. In H. Bilden \& B. Dausien (Hrsg.), Sozialisation und Geschlecht. Theoretische und methodologische Aspekte (S. 103-120). Opladen \& Farmington Hills: Verlag Barbara Budrich.

Wetterer, A. (2002). Arbeitsteilung und Geschlechterkonstruktion: "Gender at Work» in theoretischer und historischer Perspektive. Konstanz: UVK.

Schlagworte: Geschlechtersegregation am Arbeitsmarkt, geschlechtstypische Berufswahl, Berufswünsche von Frauen

\section{Entre désir et réalité: parcours d'éducation et de formation de jeunes femmes}

\section{Résumé}

Cet article contribue à examiner l'influence de personnes significatives sur la profession désirée et la profession effective des jeunes femmes. Dans cette perspective, des jeunes femmes nées entre 1983 et 1989 ont été interrogées sur leur profession rêvée et celle actuellement exercée. L'analyse de contenu a permis d'identifier quatre groupes d'acteurs significatifs qui ont exercé différentes influences sur les jeunes femmes. Les résultats révèlent un processus de sexuation, en particulier dans les familles et surtout par l'influence de la mère. Alors que les mères sont perçues comme soutien émotionnel, les pères apparaissent davantage comme une personne de contact pour le financement d'une formation continue. Des métiers typiquement féminins, tels que l'enseignement en classe maternelle ou élémentaire, sont souvent une profession rêvée dans la petite enfance; cette différenciation peut déjà être considérée comme une orientation sexuée.

Mots-clés: Discrimination de genre, sexuation, domaine professionnel, choix professionnel, souhaits professionnels des femmes

\section{Tra desiderio e realtà: corsi di formazione e percorsi professionali di donne giovani}

\section{Riassunto}

Per questo articolo sono state intervistate delle giovani donne nate tra il 1983 e il 1989 a proposito delle loro professioni ideali e delle professioni che attualmente svolgono. Attraverso un analisi qualitativa di contenuto, sono stati identificati quattro gruppi di attori significativi, che esercitano diversi tipi di influenza sulle giovani donne. I risultati mostrano che una forte marcatura di gender può essere riscontrata nelle famiglie, in particolari attraverso l'influenza della madre. Quando le madri vengono percepite come sostegno emotivo, i padri hanno 
tendenza a comparire come persone di contatto per il finanziamento della formazione continua. Alcuni mestieri tipicamente femminili, come l'insegnamento (scuola materna), sono spesso mestieri „da sogno“ in tenerà età; questa può già essere considerata come una marcatura di gender.

Parole chiave: Segregazione die generi nel mercato del lavoro, mercato del lavoro, scelta professionale con marcatura di gender, scelta professionale, desideri professionali delle donne.

\section{Between Aspiration and Reality: Educational and Professional Trajectories of Young Women}

\section{Summary}

This article aims at examining the influence of significant others on young women professional aspiration and actual profession. Young women born between 1983 and 1989 were interviewed about their dream job and their actual occupation in the present. Qualitative content analysis allowed to identify four groups of significant others who exerted different influences on the young women. Results show that the strongest gendering can be found in families, particularly via the mother's influence. While mothers are perceived as emotionally supportive, fathers tend to appear as a contact person for the funding of further education. Typically, female professions such as kindergarten teacher often appear as a dream job in early childhood, which continue the strongly gendered nature of women's educational and occupational pathways.

Keywords: Gender discrimination, gendering process, professional domain, professional choice, women's professional aspirations 
